\title{
Annalen \\ der Physik
}

(c) Johann Ambrosius Barth 1998

\section{First Order Metal-Insulator Transition in Two-dimensional Disordered Systems}

\author{
Shi-Jie Xiong ${ }^{1}$, G. N. Katomeris ${ }^{2}$ and S. N. Evangelou ${ }^{2}$ \\ ${ }^{1}$ Laboratory of Solid State Microstructures and Department of Physics, Nanjing University, \\ Nanjing 210093, China \\ ${ }^{2}$ Physics Department, University of Ioannina, Ioannina 45110, Greece
}

\begin{abstract}
In the absence of magnetic field or spin-orbit coupling the one-parameter scaling theory predicts localization of all states in two-dimensional $(2 D)$ disordered systems, for any amount of disorder. However, a $2 D$ metallic phase has been recently reported in high mobility Si-MOS and GaAs-based materials without magnetic field. We study numerically a recently proposed $2 D$ model which consists of a compactly coupled pure-random plane structure. This allows to obtain exactly a continuum of one-dimensional ballistic extended states which can lead to a marginal metallic phase of finite conductivity $\sigma_{0}=2 e^{2} / h$, in a wide energy range whose boundaries define the mobility edges of a first-order metal-insulator transition. We present numerical diagonalization results and the conductivity of the system in perpendicular magnetic field, which verify the above analytical predictions. The model is also discussed in connection to recent experiments.
\end{abstract}

Keywords: Localization, 2D Metal, Metal-insulator transition

\section{Introduction}

The research activity in the study of electronic transport in disordered mesoscopic systems has increased dramatically in recent years, following the rapid advances in microstructure technology [1]. Anderson localization of eigenstates and the associated metal-insulator transition (MIT) is the important phenomenon in this area, which is due to the presence of disorder [2]. Accordingly, a scaling theory was developed to deal with the Anderson MIT, which predicts that all states are exponentially localized in two-dimensional $(2 D)$ disordered systems [3]. Extended states with metallic diffusive regime can exist in higher than two dimensions, e.g. in weakly disordered $3 D$ systems. A dramatic experimental breakthrough in this area concerns the Si-MOSFET microstructures where a $2 D$ metallic phase was demonstrated at zero magnetic field. This unusual finding was obtained by studying the Quantum Hall Effect to insulator transition where the extended states, centered in the middle of each Landau band at high field $H$, were shown to coalesce and remain in a finite energy range as $H$

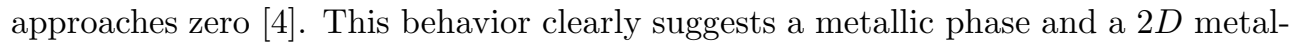
insulator transition in contradiction with the scaling theory of localization which predicts that the extended states should 'float up' in energy as $H \rightarrow 0$, leading to complete localization.

The main experimental findings can be summarized in the strong exponential drop 
of the temperature dependent resistivity at temperatures below $2 K$, in high mobility (weakly disordered) Si-MOS samples. This exponential drop is even more enhanced in higher mobility samples and can be approximated by the empirical fit [5]

$$
\rho(T) \sim \rho_{0}+\rho_{1} \exp \left(-T_{0} / T\right) .
$$

Moreover, the obtained resistivity as a function of the electron density $n$ was found to be roughly inversely proportional to the distance from the critical density $n_{c}$

$$
\rho(n) \sim \text { const. } /\left(n-n_{c}\right),
$$

with the product of the critical exponents $z \nu \approx 1$ obtained from the sample dependent temperature parameter $T_{0}$ [5], which scales as

$$
T_{0} \sim\left(n-n_{c}\right)^{z \nu} .
$$

The localization length divergence $\xi \sim\left|E-E_{c}\right|^{-\nu}$ at the mobility edge $E_{c}$ defines the localization exponent $\nu$, and the temperature dependence of the inelastic scattering length $L_{i n} \sim T^{-1 / z}$ the dynamical exponent $z$, respectively. The obtained experimental data very roughly give $\nu \approx z \approx 1$ for the two critical exponents [5]. It should be emphasised that the $2 D$ metallic phase was subsequently shown for $\mathrm{n}$ - and p-SiGe quantum wells, p-GaAs, p-AlAs and n-GaAs. Most data are rather successfully interpreted by two-parameter scaling [6], since the critical conductance varies from sample to sample, although it is rather close to the universal value $e^{2} / h$ [5].

A considerable progress has been achieved in the development of numerical techniques which have been particularly successful for studying Anderson localization. The MIT from the localized (insulating) to the extended (metallic) regime can be well understood by finite size studies, via transfer matrix products, and also matrix ensemble diagonalization, via energy level-statistical methods. It is desirable to perform a similar study for the recently proposed model of a mixed (pure-random) coupled $2 D$ system [- [], in order to demonstrate whether disorder in one sublattice only (the other is pure) still gives results in agreement with the predictions of the scaling theory for $2 D$. In this system we shall derive a continuous $1 D$ manifold of ballistic extended states emerging, within the majority of localized states. The presence of this manifold of extended states will be also confirmed by numerical studies of the corresponding tight-binding Hamiltonian in the presence of disorder $W$ in one sublattice and interlattice coupling $t$. The localization properties of the system will be studied by the participation ratio of the corresponding eigenstates. Our aim is to show that such mixed structures can give rise to extended states, which are required in order to obtain a metallic phase in $2 D$ disordered systems. The obtained results are surprisingly close to the experimental data for Si-MOS, which indicate some kind of a first-order MIT [8].

The paper is organized as follows: Section II gives an overview of the model and the physical parameters characterizing the proposed mixed system. In Section III we derive the $1 D$ extended states. The density of states and the eigenstate behavior of the model is studied by numerical diagonalization in Section IV, whereas the properties of the conductance in perpendicular magnetic field in Section V. Finally, in the last section we give a summary of our conclusions. 


\section{The model}

Although the one-parameter scaling theory predicts always localization in one or two dimensions, isolated extended states can exist even in $1 D$ as a consequence of specific short-range disorder correlations [9, 10]. In $2 D$ disordered systems delocalization is possible for noninteracting electrons only by breaking the time-reversal symmetry, via a magnetic field, or the spin-rotation invariance, by spin-orbit coupling [11]. In continuous $2 D$ models extended states were previously shown in a model with random $\delta$-function potentials of infinitesimal action distance [12]. The presence of extended states in $2 D$ random systems is undoubtetly interesting in connection with the recently reported metallic phase and the MIT in Si-MOS structures and GaAs-based materials, in the absence of magnetic fields [5].

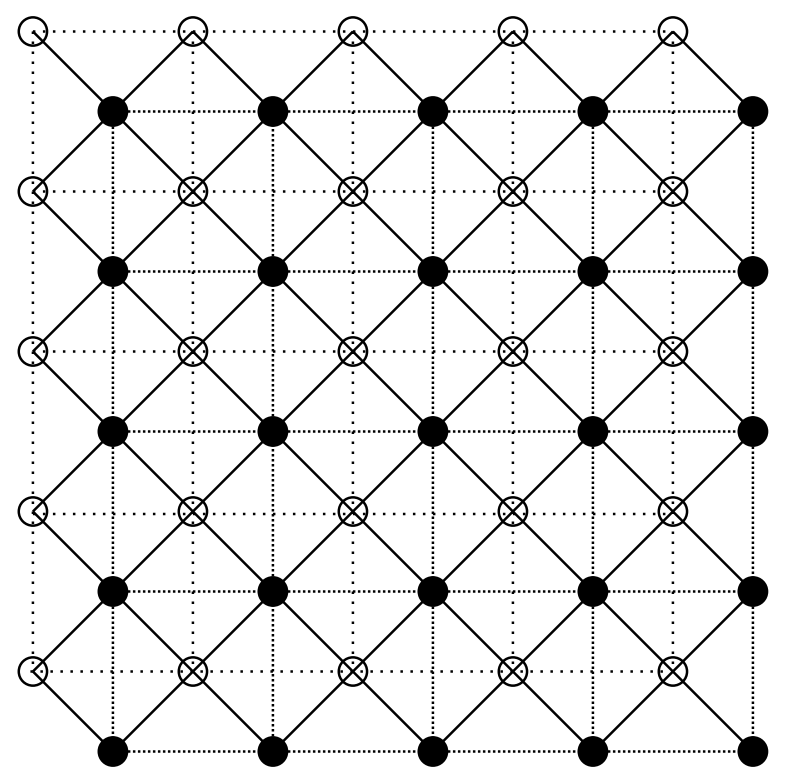

Fig. 1: The studied $2 D$ pure-random coupled structure for $L=10$. The $L^{2} / 4$ open circles denote the pure lattice sites with disorder $W=0$ and the $L^{2} / 4$ closed circles the random lattice sites with finite disorder $W$. The two sublattices are coupled by hopping matrix elements $t$.

We consider the $2 D$ random system shown in Fig. 1, which is composed of two compactly coupled square lattices, one random and the other periodic. The sites of one lattice are located above the centers of the plaquettes of the other. The pure plane has zero site disorder and the random plane has finite site disorder estimated by $W$, while the coupling between the two planes is denoted by $t$. We shall demonstrate analytically that extended wavefunctions can exist in the considered partially random structure. These are perfectly extended (ballistic) states with a special momentum and form a $1 D$ continuum, which gives a finite $2 e^{2} / h$ conductivity in a specific energy range with its boundaries defining the mobility edges (ME) of a first-order MIT. Moreover, they can coexist with localized states, as expected from the scaling theory and under long-range Coulomb interactions the system can reduce [7] to the marginal Fermi liquid proposed in 13]. 
The corresponding tight-binding Hamiltonian reads

$$
H=H_{p}+H_{r}+H_{c}
$$

where $H_{p}, H_{r}$ and $H_{c}$ denote the parts corresponding to pure, random and the coupling between the two sublattices, respectively. For convenience, the length units and the origin of the coordinates are set so that the pure (random) sites count odd (even) numbers, e.g. the odd indexes $(n, m)$ denote a pure lattice site and even indexes $(n, m)$ a random lattice site, respectively. The Hamiltonian in the $2 D$ coordinates $(n, m)$ can be written as

$$
H_{p}=\sum_{n, m=o d d}\left(a_{n, m}^{\dagger} a_{n+2, m}+a_{n, m}^{\dagger} a_{n-2, m}+a_{n, m}^{\dagger} a_{n, m+2}+a_{n, m}^{\dagger} a_{n, m-2}\right),
$$

and

$$
\begin{aligned}
H_{r} & =\sum_{n, m=\text { even }} \epsilon_{n, m} a_{n, m}^{\dagger} a_{n, m} \\
& +\sum_{n, m=\text { even }}\left(a_{n, m}^{\dagger} a_{n+2, m}+a_{n, m}^{\dagger} a_{n-2, m}+a_{n, m}^{\dagger} a_{n, m+2}+a_{n, m}^{\dagger} a_{n, m-2}\right),
\end{aligned}
$$

with the coupling between them

$$
H_{c}=t \sum_{n, m}\left(a_{n, m}^{\dagger} a_{n+1, m+1}+a_{n, m}^{\dagger} a_{n-1, m+1}+a_{n, m}^{\dagger} a_{n+1, m-1}+a_{n, m}^{\dagger} a_{n-1, m-1}\right)(7)
$$

where $a_{n, m}$ is the destruction operator for an electron at the site $(n, m)$ of the pure plane $(\mathrm{n}, \mathrm{m}=\mathrm{odd})$ or the random plane $\left(\mathrm{n}, \mathrm{m}=\right.$ even). The coupling Hamiltonian $H_{c}$ connects each pure (random) site to its four nearest-neighbor random (pure) sites via the hopping parameter $t$. The site energies in the random plane $\epsilon_{n, m}$ are from a uniform distribution between $-W / 2$ and $W / 2$.

\section{Extended states in the $2 D$ random structure}

Extended wave functions can have zero amplitude on the random sites and finite amplitude on the pure sites. This requirement leads to the equation

$$
t(\psi(n, m)+\psi(n+2, m)+\psi(n, m+2)+\psi(n+2, m+2))=0,
$$

for the wavefunction amplitudes $\psi$ on the four pure lattice sites with odd $(n, m)$, $(n+2, m),(n, m+2)$ and $(n+2, m+2)$ which are adjacent to the random lattice site $(n+1, m+1)$ having zero amplitude. This equation is satisfied by the pure plane wave states $\psi_{k_{x}, k_{y}}(n, m) \sim \exp \left(i k_{x} n+i k_{y} m\right)$, which leads to the condition

$$
\left(1+\exp \left(i 2 k_{x}\right)\left(1+\exp \left(i 2 k_{y}\right)\right)=0,\right.
$$

that is $k_{x}=\pi / 2$, or $k_{y}=\pi / 2$. Therefore, we can immediately write down extended states which are completely unaffected by the randomness in the considered $L \times L$ structure, with periodic boundary conditions in all directions. In order to obtain such states for finite $L$ with the adopted boundary conditions, $L$ must be even and multiple 
of four so that $k_{x}=\pi / 2$ or $k_{y}=\pi / 2$ denote eigenstates of the pure plane. They take the normalized form

$$
\begin{aligned}
& \psi_{1, k_{x}}(n, m)=\frac{2}{L} \sin \left(\frac{m \pi}{2}\right) \exp \left(i k_{x} n\right), \\
& \psi_{2, k_{y}}(n, m)=\frac{2}{L} \sin \left(\frac{n \pi}{2}\right) \exp \left(i k_{y} m\right),
\end{aligned}
$$

where $n, m$ are the odd site coordinates of the pure lattice and $k_{x}\left(k_{y}\right)=2 j_{x}\left(j_{y}\right) \pi / L$, $j_{x}\left(j_{y}\right)=1,2, \ldots, L / 2$. The states $\psi_{1, k_{x}}(n, m), \psi_{2, k_{y}}(n, m)$ are exact eigenstates of the Hamiltonian $H$ which survive in the considered mixed pure-random coupled structure as it can be easily verified by applying $H$ on $\psi_{1, k_{x}}(n, m), \psi_{2, k_{y}}(n, m)$. One obtains the corresponding eigenenergies of these states

$$
E=2\left[\cos \left(2 k_{x}\right)-1\right], E=2\left[\cos \left(2 k_{y}\right)-1\right],
$$

respectively. Since their transverse momentum is fixed to $k_{y}\left(k_{x}\right)=\pi / 2$ while their longitudinal momentum $k_{x}\left(k_{y}\right)$ runs in the $1 D$ Brillouin zone, they form a $1 D$ continuum in the energy range $[-4,0]$ [7].

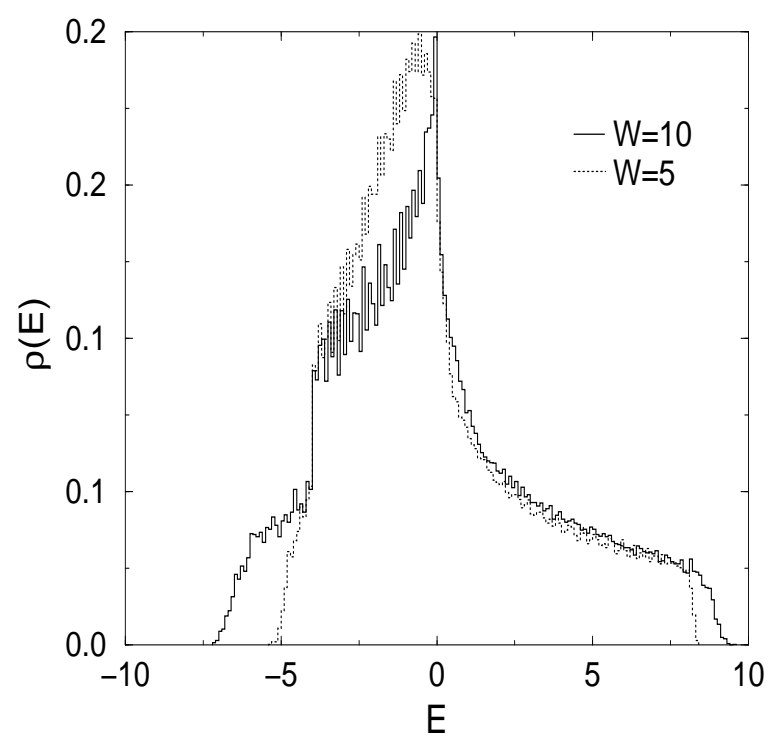

Fig. 2: The density of states $\rho(E)$, in arbitrary units, for the considered $2 D$ random structure with linear size $L=100$ with $t=1$, by taking average over 8 random configurations. It the region $[-4,0]$ the continuous manifold of pure extended states coexists with other localized states.

The perfectly extended states found have momentum along both the $2 D$ principal axes, $x$ (or $y$ ) direction, independently of the system size $L$. Moreover, they do not violate the scaling theory since they effectively decouple from the random lattice although the two sublattices remain compactly coupled. They lead to minimum metallic conductivity $\sigma_{0}=2 e^{2} / h$ (if spin is included) and one obtains a first-order MIT from $\sigma_{0}$ to 0 at $E_{c 1}=-4$ and $E_{c 2}=0$, which can be regarded as mobility 
edges [7]. The rest of the $2 D$ states are asymptotically localized, as expected from the scaling theory [3]. We also observe that the lower ME at $E_{c 1}=-4$ lies near the bottom of the band so that the Fermi energy can be moved into the metallic regime even for small electronic doping, in agreement with silicon MOSFETs where the metallic phase is achieved at very low electron densities $\left(\sim 10^{11} \mathrm{~cm}^{-2}\right)[$ [. It should be also noted that a minimum metallic conductivity around $e^{2} / h$ has been suggested from experimental data for holes in GaAs heterostructures [5].

We should also notice that the obtained $\sigma=\sigma_{0}$ in the energy window $\left[E_{c 1}, E_{c 2}\right]$ is scale invariant, so that the system does not reache a true metallic phase but a critical one instead. The corresponding $\beta$-function becomes zero and not positive in the considered $2 D$ system [3]. Moreover, the obtained extended states which coexist with the localized states in the above wide energy range, have lower dimensionality and measure, so that in ref. [7] the obtained phase was renamed "marginal metallic phase", adopting the terminology "marginal Fermi liquid" proposed by Varma el al [13.

\section{Numerical diagonalization}

We have performed exact numerical diagonalization of the $L \times L(L / 2 \times L / 2$ pure and $L / 2 \times L / 2$ random) system adopting periodic boundary conditions in the two directions. We obtain $L / 2+L / 2-1=L-1$ perfect $1 D$ extended electronic states among the rest of localized states in [-4,0], out of the total number of $L^{2} / 2$ states. In Fig. 2 we show the obtained density of states for two values of the disorder $W$. It is seen that the characteristic logarithmic spectral singularity of the pure lattice at $E=0$ in two dimensions is recovered for large disorder $W$. This is due to the fact that by increasing $W$ more and more states aquire very large localization lengths, since the electrons avoid altogether the random lattice and the pure lattice eventually decouples from the system for $W \rightarrow \infty$.

We have also verified by transfer matrix studies that the smallest Lyapunov exponent which corresponds to the inverse localization length, is exactly zero for any $W$ in the extended energy window $[-4,0]$. This calculation which confirms the presence

of the $1 D$ extended states will be presented elsewhere. However, from the numerical diagonalization we can compute the participation ratio of each eigenstate, via

$$
P=\left(\sum_{n, m} \Psi_{n, m}^{4}\right)^{-1},
$$

which gives a measure of the effective number of sites occupied by the eigenstate. $P \propto L^{2}$ for a perfectly extended eigenstate and $P \propto$ const. for a localized state. Fig. 3 shows the $1 D$ extended states which have large $P$ values extending over all the pure lattice. We show the participation of the rest of states in the pure (white dots) and the random (black dots) sublattices. The participation is also large for the rest of states, despite the fact that the disorder choice $W=10$ is the most favourable for localization in the system. We have shown that the majority of the rest of states have very large localization lengths and are critical in nature, as expected in $2 D$.

\section{Perpendicular magnetic field}

We have also computed the change of the conductance in the presence of a perpendicular magnetic field which is known to destroy the extended states [5]. The conductance 


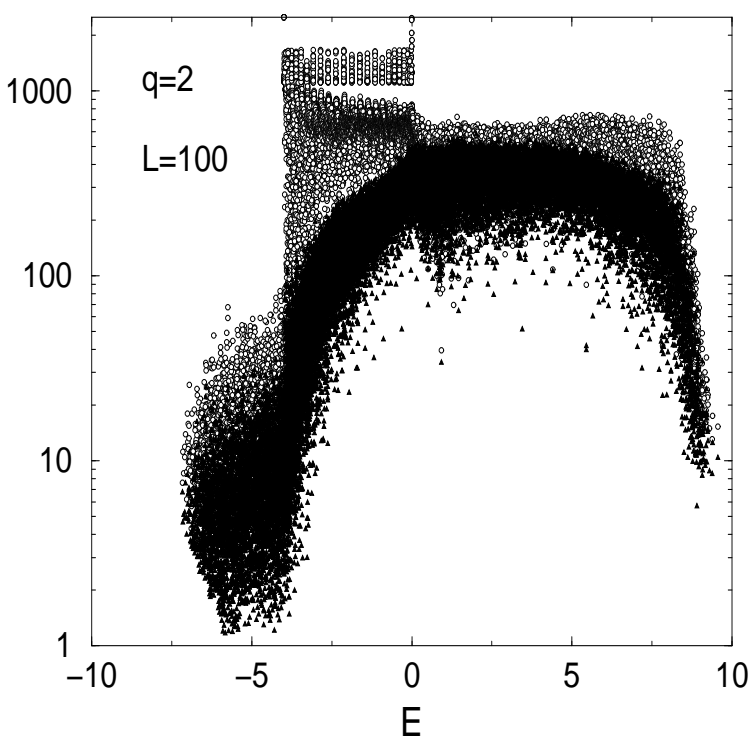

Fig. 3: The participation ratio $P$ (from the second $q=2$ moment of the amplitudes from Eq. (13)) versus energy for a fixed size $L=100$ system with $W=10$ and $t=1$ by presenting data for 8 random configurations. The $1 D$ manifold of extended states is clearly seen in the upper part of the figure within the energy range $[-4,0]$. The white(black) dots give the participation (extend) of each state on the pure(random) sublattice.

$\sigma$ equals the conductivity for a square sample in $2 D$ and can be obtained from the multichannel Landauer-Büttiker formula for a $2 D$ system of size $L$ via

$$
\sigma(L)=\left(e^{2} / h\right) \operatorname{Tr}\left[T^{+}(L) T(L)\right],
$$

where $T(L)$ is the $L$-channel transmission matrix for electronic propagation. We adopt the Coulomb gauge for the perpendicular magnetic field and show the obtained results in Figs. 4 and 5. They demonstrate the reduction of the conductance in the presence of a perpendicular magnetic field.

\section{Conclusions}

There have been a few theoretical attempts in order to understand the experimental puzzle of the $2 D$ metallic phase, such as scaling in the presence of interactions [14], via hole traps [15], two-phase models [16], and superconductivity [17], etc. The basic mechanism was attributed to Coulomb interactions which leads to violation of the scaling theory and the presence of a metallic phase in $2 D$. However, these approaches suffer from serious experimental problems (e.g. the transition to Wigner solid, no traps or two phases exist for GaAs, no superconducting $T_{c}$, etc.) In this paper we present a simple transparent alternative of the $2 D$ metallic phase via a first order MIT from the localized $2 D$ regime to an extended ballistic regime, which coexists with the localized phase. For this purpose we discuss an explicitly solved model of a pure-random mixed structure which can describe the Si-MOSFET under certain 


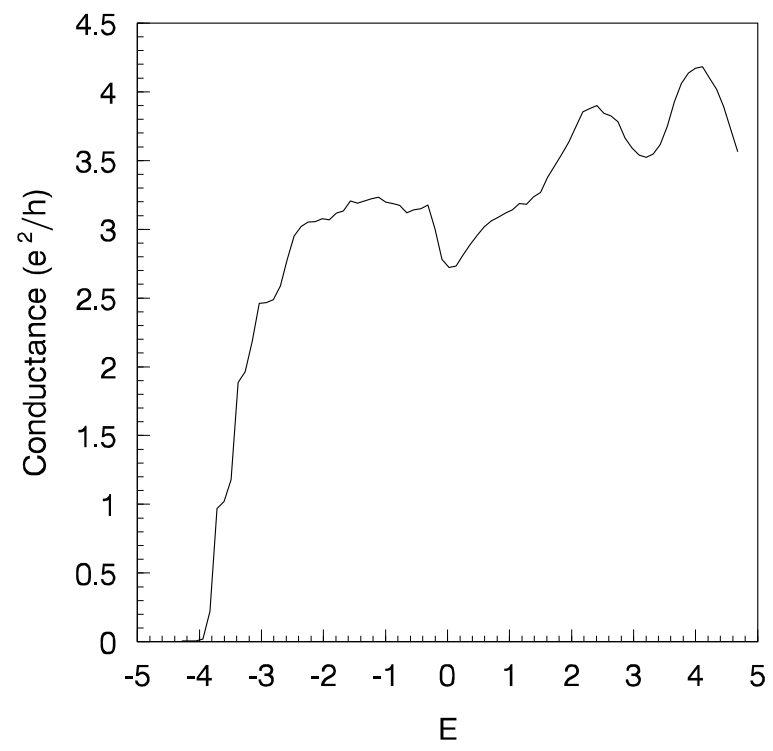

Fig. 4: The "metallic" region energy dependence of the conductance in a perpendicular magnetic field of strength of $0.1 \phi_{0}$ per square plaquette, where $\phi_{0}$ is the flux quantum and the fixed system size $L=24$. The interlattice hopping is $t=1.2$ and the disorder in the random sublattice is $W=8$.

assumptions. We determine exactly the band of $1 D$ ballistic extended states which propagate freely in the $2 D$ mixed pure-random system, without magnetic field or spinorbit coupling. These extended states are located at the edges of the first Brillouin zone $\left(k_{x}=\pi / 2\right.$, any $k_{y}$ or $k_{y}=\pi / 2$, any $\left.k_{x}\right)$ and lie in an energy regime whose boundaries define the first-order MIT. We perform numerical computation for the complete eigensolutions of the corresponding random Hamiltonian matrix ensemble and also present the conductance as a function of a perpendicular magnetic field.

The extended electrons found form a pseudo-Fermi sea embedded in the localized electrons and the system can be regarded as a $2 D$ non-Fermi liquid because of its measure, the $1 D$-like character of the spectrum, as well as the presence of a background from the localized electrons. The pseudo-Fermi surface has only four points corresponding to two Luttinger liquids crossing with each other [7]. Our results are important for understanding many experimental findings related to the $2 D$ MIT in Si MOSFETs and GaAs-AlGaAs heterostructures. In these systems the electrons or holes are confined within several atomic planes and due to lattice mismatch, or atomic diffusion, it could be expected that the planes adjacent to the interface are random, while some planes relatively far from the interface are less random and, as we have shown, could support ballistic channels. Although for these materials more realistic models are certainly needed our results may still shed some light on the important phenomenon of the $2 D$ metallic phase.

This work was supported in part by TMR and also a IENE $\Delta$ Research Grant of the Greek Secretariat of Science and Technology. 


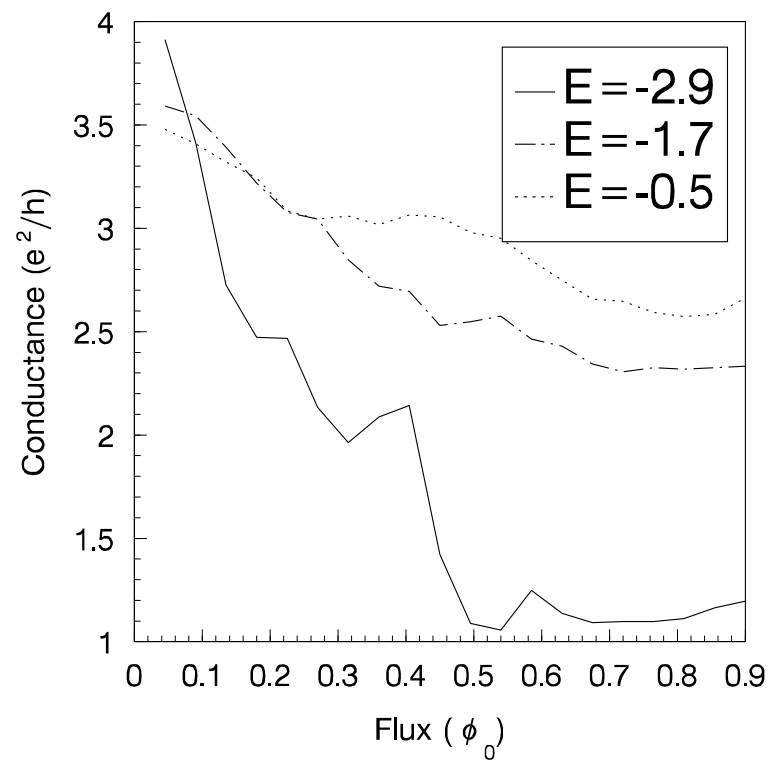

Fig. 5: The magnetic field dependence of the conductance for various energies in the "metallic" regime with the rest of the parameters the same as in Fig.4. We observe that the conductance drops when the magnetic field is introduced, in agreement with the experimental findings.

\section{References}

[1] Mesoscopic Phenomena in Solids, B.L. Altshuler, P. A. Lee, and R.A. Webb Eds., North Holland, Amsterdam, 1991; for a review see P.A. Lee and T.V. Ramakhrishnan, Rev. Mod. Phys. 57, 28 (1985).

[2] P.W. Anderson, Phys. Rev. 1091492 (1958).

[3] E. Abrahams, P.W. Anderson, D.C. Licciardello, and T.V. Ramakrishnan, Phys. Rev. Lett. 42, 673 (1979).

[4] S.V. Kravchenko, W.E. Mason, J.E. Furneaux, and V.M. Pudalov, Phys. Rev. Lett. 75, 910 (1995).

[5] S. V. Kravchenko, G. V. Kravchenko, J. E. Furneaux, V. M. Pudalov and M. D'Iorio, Phys. Rev. B 50, 8039 (1994); S. V. Kravchenko, W. E. Mason, G. E. Bowkev, J. E. Furneaux, V. M. Pudalov and M. D'Iorio, Phys. Rev. B 51, 7038 (1995); S. V. Kravchenko, D. Simonian, M. P. Savacchik, W. Mason and J. E. Furneaux, Phys. Rev. Lett, 77, 4938 (1996); Y. Hanein, U. Meirav, D. Shahar, C.C. Li, D.C. Tsui and Hadas Shtrikman, preprint, cond-mat/9709184; D. Simonian, S.V. Kravchenko, and M.P. Sarachik, preprint, cond-mat/9801077 D. Simonian, S.V. Kravchenko, M.P. Sarachik and V.M. Pudalov, Phys. Rev. Lett. 79, 2304 (1997); cond-mat/9801077;M.Y. Simmons, A.R. Hamilton, M. Pepper, E.H. Linfield, P.D. Rose, and D.A. Ritchie, preprint, cond-mat/9709240; Y. Hanein, U. Meirav, D. Shahar, C.C. Li, D.C. Tsui and Hadas Shtrikman, preprint, cond-mat/9709184.

[6] N.-J. Kim, D. Popovic and S. Washburn, preprint, cond-mat/9809357.

[7] S.-J. Xiong, S.N. Evangelou and E.N. Economou, preprint, cond-mat/9807162.

[8] V. Dobrosavljević, E. Abrahams, E. Miranda, and S. Chakravarty, Phys. Rev. Lett. 79, 455 (1997). (1989). 
[9] D.H. Dunlap, H-L. Wu and P.W. Phillips, Phys. Rev. Lett. 65, 88 (1990).

[10] S. J. Xiong, Y. Chen and S. N. Evangelou, Phys. Rev. Lett., 77, 4414 (1996).

[11] S. N. Evangelou and T. Ziman, J. Phys. C 20, L235 (1987); T. Ando, Phys. Rev. B 40, 5325 (1989); S. N. Evangelou, Phys. Rev . Lett 75, 2550 (1995); T. Kawarabayashi and T. Ohtsuki, Phys. Rev. B. 53, 6975 (1996).

[12] M. Ya. Azbel, Phys. Rev. B 45, 4208 (1992).

[13] C. M. Varma, P. B. Littlewood, S. Schmitt-Rink, E. Abrahams, and A. E. Ruckenstein, Phys. Rev. Lett. 63, (1996).

[14] A.M. Filkelstein, Zh.E.T.F. 84, 168 (1983) [JETP 97, 83 (1983)]; JETP Lett. 40, 63 (1984); Castelani et al., Phys. Rev. B 57, 9381 (1998).

[15] B. L. Altshuler and D. Maslov, preprint, cond-mat/9805383.

[16] S. He and X.C. Xie, Phys. Rev. Lett. 80, 3324 (1998).

[17] P. Phillips and Y. Wang, preprint, cond-mat/9704200. 